\title{
A rare case of urothelial carcinoma arising in mature cystic teratoma
}

\author{
Nitesh R. Maurya ${ }^{1 *}$, Urvi C. Bhavsar ${ }^{2}$ \\ ${ }^{1}$ Department of Surgical Oncology, Sri Shankara Cancer Hospital and Research Centre, Bangalore, Karnataka, India
}

${ }^{2}$ Saurabh Clinic, Thane, Mumbai, Maharastra, India

Received: 22 April 2018

Accepted: 23 May 2018

\section{*Correspondence:}

Dr. Nitesh R. Maurya,

E-mail: nitez.23@gmail.com

Copyright: $(\odot$ the author(s), publisher and licensee Medip Academy. This is an open-access article distributed under the terms of the Creative Commons Attribution Non-Commercial License, which permits unrestricted non-commercial use, distribution, and reproduction in any medium, provided the original work is properly cited.

\begin{abstract}
Mature cystic teratoma is a common benign adnexal tumour in females. It is composed of all the three germ layers (mesoderm, endoderm and ectoderm). Malignant transformation in a mature cystic teratoma of the ovary is rare, occurring in only $1-2 \%$ of cases. The most common malignancy is squamous cell carcinoma, which consists of about $80 \%$ of malignant transformations. Here we report malignant transformation of MCT in a 74year old post-menopausal female. Grossly the ovary was large cystic occupying the entire abdomen. Trial dissection was done and only $50 \%$ of the mass could be dissected due to gross adhesions. HPE reported as urothelial carcinoma in mature cystic teratoma. MCT is easily diagnosed via sonography, but malignant transformation is difficult to identify before surgery. For patients with malignant transformation, squamous cell carcinoma is the most common malignancy arising from MCT, and accounts for $80 \%$ of all cases. Other malignancies, such as adenocarcinomas, sarcomas or malignant melanomas have been reported; however, urothelial carcinoma arising from MCT is rare.
\end{abstract}

Keywords: Germ cell tumors, Mature cystic teratoma, Urothelial carcinoma

\section{INTRODUCTION}

Mature cystic teratoma (MCT) is a common benign adnexal tumour in females in the reproductive age group. ${ }^{1,2}$ Malignant transformation in mature cystic teratoma of the ovary is rare, occurring in only $1-2 \%$ of cases. $^{1,2}$ The most common malignancy is squamous cell carcinoma, which consists of about $80 \%$ of malignant transformations. ${ }^{1}$ Urothelial carcinoma in mature cystic teratoma is very rare and occurs in $1-2 \%$ of cases. ${ }^{2}$ The most common presentation in malignant transformation of mature cystic teratoma is increased abdominal girth associated with or without abdominal pain. ${ }^{3}$

\section{CASE REPORT}

Here we report malignant transformation of MCT in a 74year old post-menopausal female. She presented to the outpatient clinic with complains of abdominal distension for 6 months associated with pain. On per abdominal examination there was a large, globular, non-mobile and tender mass extending up to the epigastrium. CT scan of the abdomen revealed a $18 \mathrm{cms} * 14 \mathrm{cms} * 13 \mathrm{cms}$ multiloculated cystic mass with thick septations. The mass originated from the pelvis and extended up to the abdomen. Ca 125 was $400 \mathrm{u} / \mathrm{ml}$. All other blood investigations were within normal limits.

The patient was planned for exploratory laprotomy. Intraoperatively there was a large tense cystic mass occupying the entire abdomen. Small bowel loops were adherent all around with large veins coursing over the cyst. Posteriorly the cyst was adherent to the posterior abdominal wall and infiltrating the distal root of mesentery. Urinary bladder was densely adherent. Attempts at releasing the bowel adhesions from the cyst led to bleeding. The cyst was opened and approximately 
2.2 litres of brownish fluid was aspirated. The cyst also had hair, fat and cartilage. 50\% of the cyst wall was excised and sent for frozen section along with part of omentum. The frozen section reported as benign teratoma with cystic fluid and omentum negative for malignancy. In view of continuous bleeding during attempts to resect the mass, the procedure was abandoned. The postoperative period was uneventful.

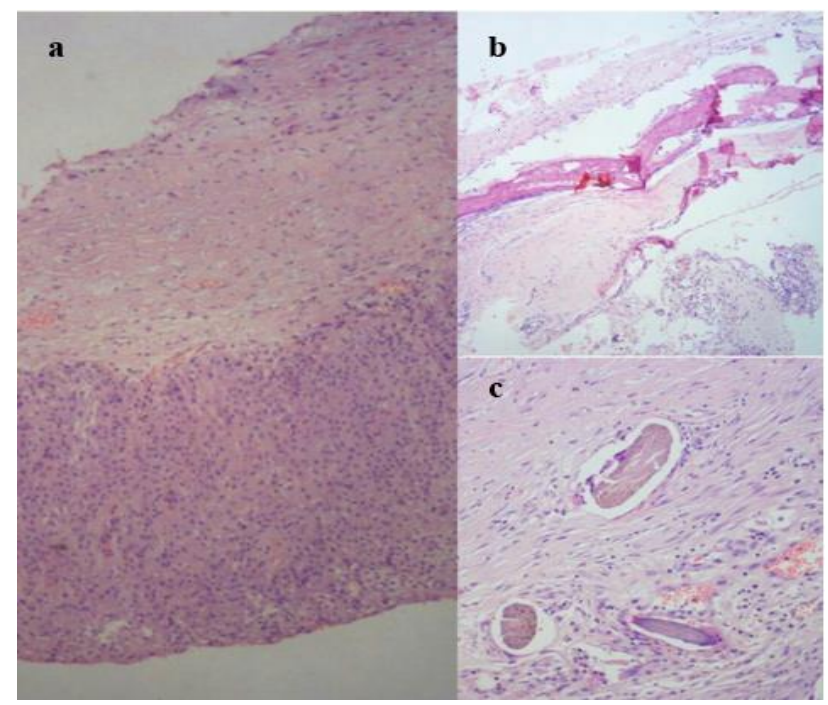

Figure 1: (a) Microscopic features showing low grade urothelial carcinoma. (b) High power microscopic feature of bone and (c) hair follicle.

Microscopically, the cystic mass had components of low grade non-invasive urothelial carcinoma (Figure 1a) along with components of bone (Figure 1b) and hair follicles (Figure 1c). The ovarian surface and capsule were free of tumor. Immuno-histochemistry was positive for CK20, CK7 and throbomodulin and negative for WT1. These results were consistent with urothelial carcinoma arising in mature cystic teratoma.

The case was discussed in multi-disciplinary tumor board and it was decided to follow up the patient at 3 monthly intervals. Chemotherapy was deferred due to large residual mass and low grade of tumour. On follow up at 2 months the patient had an episode of bleeding per rectum. On investigating her with MRI, it revealed a geographic lesion with eccentric nodular wall thickening in continuity/ infiltrating the recto- sigmoid junction (Figure 2a). It was seen encompassing the fundus and the upper body of the uterus.

It was abutting the posterior wall of the urinary bladder with indistinct fat planes. There was no evidence of proximal bowel obstruction. Colonoscopy revealed an ulcerated cystic lesion communicating with sigmoid colon and compressing it. Biopsy from the lesion revealed a low grade invasive urothelial carcinoma (Figure 2b). Patient was given hemostatic radiation and treated symptomatically. The patient eventually died of extensive disease and sigmoid infiltration after 4 months.

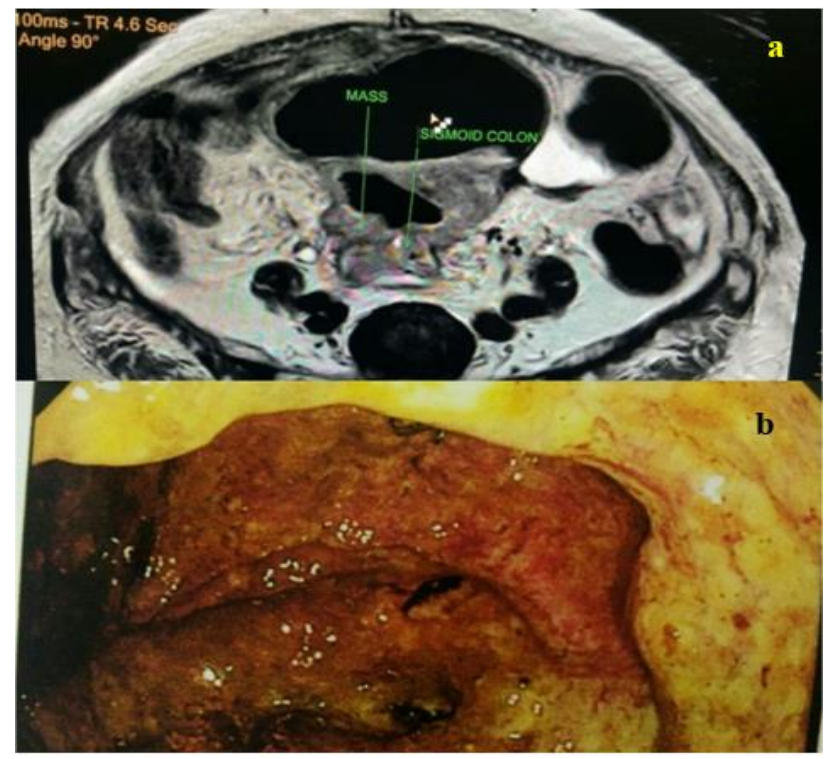

Figure 2: (a) MRI of abdomen and pelvis showing an eccentric mass infiltrating the sigmoid colon. (b) Colonoscopy showing mass infiltrating the sigmoid colon.

\section{DISCUSSION}

MCT is one of the most common germ cell ovarian tumor and accounts for $10-25 \%$ of ovarian tumours. ${ }^{1}$ It is generally seen in pre-menopausal females. Malignant transformation is rare and is generally seen in postmenopausal age group. ${ }^{2}$ The incidence of malignant transformation is $1-2 \%{ }^{1,2}$ Squamous cell carcinoma is most common malignant transformation and accounts for $80 \%$ of the cases. ${ }^{2}$ Historically these tumors are diagnosed postoperatively and are often present as an incidental finding histologically. Urothelial carcinoma (UC) in MCT is rare and very few cases have been reported in literature so far. Because of its rarity we report this case and review the literature regarding the pathogenesis and management of urothelial carcinoma arising in mature cystic teratoma.

The pathogenesis of urothelial carcinoma is not fully established yet. Lee et al found normal urothelial cells adjacent to urothelial carcinoma and they were of the opinion that secondary malignancy was induced due to prolonged stimulation of epithelial cells by lipid material. $^{2}$ The most common presenting symptom was abdominal distension and/or abdominal pain. ${ }^{3-5}$ Chuang $\mathrm{HY}$ et al in their study reviewed 5 cases of UC in MCT including theirs and concluded that malignant transformation occurs in post-menopausal age group and the average age at diagnosis is 53 years. ${ }^{1}$ In our case, patient was 72 years old. The average size of tumour in all the 5 cases were $>10 \mathrm{cms}$. In our case the tumour size was $14 \mathrm{cms}$. Although malignant transformation can occur in any age group, it is commonly seen in postmenopausal females. ${ }^{1,2}$ The other high-risk factors include age $>45$ years, elevated serum markers like $\mathrm{Ca}-$ 
125, Ca19-9, CEA, squamous cell carcinoma antigen and size more than $10 \mathrm{cms}^{3,4}$ Though Ca-125 and Ca19-9 are elevated in most of these cases it cannot be used to screen or diagnose malignant transformation in MCT especially urothelial carcinoma.

Chuang HY et al also discussed imaging modalities in diagnosis of malignancy in MCT and were of the opinion that MRI is useful in the diagnosis of occult malignancy in MCT. ${ }^{1,3,7}$ The Rokitansky nodule is a pathognomic feature of MCT. The presence of transmural growth pattern in Rokitansky nodule favors malignancy. ${ }^{3}$ MCT with malignant transformation are characterized by specific MRI findings: size $>10 \mathrm{~cm}$, transmural or extracapsular growth with extension into adjacent structures, presence of solid and enhancing soft tissue component. ${ }^{3}$ In our case MRI showed infiltration of recto-sigmoid junction with tumour mass which was confirmed on colonoscopy and guided biopsy.

Immuno-histochemistry serves as an important diagnostic tool in differentiating UC arising in MCT from Transitional cell carcinoma of ovary (TCC-O). ${ }^{6}$ Ok-Jun Lee in their study found most UC of urinary tract were positive for CK-7, CK-20, thrombomodulin and negative for WT-1. ${ }^{2}$ TCC-O on the other hand was positive for WT-1. In our case immune-histochemistry was positive for CK-7, CK-20, thrombomodulin and negative for WT1 favouring the diagnosis of UC arising from urothelial cells.

As urothelial carcinoma is rare there is no fixed consensus on treatment protocols. Several studies concluded that surgical staging remains the mainstay of treatment. $^{1,2}$ Further treatment depends upon the grade and stage of the disease. Early stage disease can be managed conservatively with regular follow up while patients with advanced stage disease require adjuvant chemotherapy and radiation. ${ }^{1,2}$ In our case after debulking surgery, patient was kept on follow-up. Chemotherapy was deferred in view of large residual mass and lowgrade nature of the tumours.

\section{CONCLUSION}

$\mathrm{UC}$ is a very rare form of secondary neoplasm arising in MCT. A malignant transformation should be suspected for patients with large tumour size, post-menopausal age group, and MRI showing transmural enhancing soft tissue component in Rokitansky nodule. Proper surgical staging should be carried out and depending on the grade and stage of the disease follow up or adjuvant treatment should be planned.

Funding: No funding sources

Conflict of interest: None declared

Ethical approval: Not required

\section{REFERENCES}

1. Chuang HY, Chen YT, Mac TL, Chen YC, Chen HS, Wang WS et al. Urothelial carcinoma arising from an ovarian mature cystic teratoma. Taiwanese J Obstet Gynecol. 2015;54:442-4.

2. Lee OJ, Lee HC. Urothelial (transitional cell) carcinoma arising in mature cystic teratoma: A case report. Korean J Pathol. 2010;44:666-9.

3. Park SB, Kim JK, Kim KR Cho KS. Preoperative diagnosis of mature cystic teratoma with malignant transformation: analysis of imaging findings and clinical laboratory data. Arch Gynecol Obstet. 2007;275:25-31.

4. Al-Rayyan ES, Duqoum WJ, Sawalha MS, Nascimento MC, Pather S, Dalrymple CJ, et al. Secondary malignancies in ovarian dermoid cyst. Saudi Med J. 2009;30:524-8.

5. Hackethal A, Brueggmann D, Bohlmann MK, Franke FE, Tinneberg HR, Münstedt K, et al. Squamous cell carcinoma in mature cystic teratoma of ovary: systematic review and analysis of published data. Lncet Oncol. 2008;9:1173-80.

6. Eichhorn JH, Young RH. Transitional cell carcinoma of ovary and a morphologic study of 100 cases with emphasis on differential. Am j Surg Pathol. 2004;28:453-63.

7. Kido A, Togashi K, Konishi I, Kataoka ML, Koyama $\mathrm{T}$, Ueda $\mathrm{H}$, et al. Dermoid cyst of the ovary with malignant transformation: MR appearance. AJR Am J Roentgenol. 1999;172:445-9.

Cite this article as: Maurya NR, Bhavsar UC. A rare case of urothelial carcinoma arising in mature cystic teratoma. Int J Reprod Contracept Obstet Gynecol 2018;7:2959-61. 